Brazilian Journal

of Chemical

Engineering

\title{
CONTROLLED RELEASE OF THEOPHYLLINE- CHITOSAN COMPOSITE PARTICLES PREPARED USING SUPERCRITICAL ASSISTED ATOMIZATION
}

\author{
Hsien-Tsung $\mathrm{Wu}^{1 *}$, Hou-Cyuan Chen ${ }^{1}$ and Hsiao-Kang Lee ${ }^{1}$ \\ ${ }^{1}$ Ming Chi University of Technology, Department of Chemical Engineering, New Taipei City 24301, Taiwan. \\ E-mail: stwu@mail.mcut.edu.tw - ORCID: 0000-0001-5594-0444 \\ (Submitted: April 11, 2018 ; Revised: September 18, 2018 ; Accepted: September 26, 2018)
}

\begin{abstract}
This study investigated the formation of composite particles of chitosan (CS) and theophylline (TPH) via supercritical assisted atomization (SAA) using aqueous ethanol $(50 \%, \mathrm{v} / \mathrm{v})$ as the solvent and supercritical $\mathrm{CO}_{2}$ as the spraying medium. According to XRD, DSC, and FTIR analyses, the crystal form of the SAA-treated TPH from the as-received TPH was unchanged. The effect of different mass ratios of CS to TPH on the in vitro release of TPH showed that the dissolution of the highly water-soluble TPH was retarded when included in the composite particles and could be controlled by the mass ratio of the component in the SAA process. The in vitro dissolution data showed a good fit with the Peppas-Sahlin model, which was used to conclude that the drug release from the composite particles resulted from a combination of drug diffusion and relaxation of the polymer. As the mass ratio of CS to TPH increased, the mechanism relating to polymer relaxation became crucial in the TPH-CS composite particles.

Keywords: Supercritical assisted atomization; Theophylline; Chitosan; Composite particles; Controlled release.
\end{abstract}

\section{INTRODUCTION}

Theophylline (TPH) is widely used as a bronchodilator in asthma therapy and is usually administered in tablet form (Nunthanid et al., 2004) or in solutions. TPH has been reported to exist in both a monohydrate and an anhydrous form. Anhydrous TPH has two polymorphic forms, i.e., stable form I and metastable form II (Legendre and Randzio, 2007). Drug with different particle sizes can be administered as dry powder for inhalation administration, as suspension for parenteral routes, and as capsules or tablets for oral routes. The prevention of the symptoms of chronic asthma has been demonstrated when serum theophylline concentrations are maintained at levels within the $10-20 \mu \mathrm{g} / \mathrm{ml}$ therapeutic range (Hendeles et al., 1987). To avoid adverse effects, the combination of a biopolymeric matrix with the drugs to control their release has become important in the development of sustained-release dosage types (Nunthanid et al.,
2004; Talukdar et al., 1998). Hydrophilic carriers with enhanced dissolution properties (for example, polyvinylpyrrolidone, hydroxypropyl cellulose, and polyethylene glycol) should not be used with BCS class I theophylline (high solubility and permeability) (Lindenberg et al., 2004) because the burst effect should be avoided.

Chitosan (CS) is a partially N-deacetylated derivative of chitin, which is commonly found in insect and crustacean shells, as well as cell walls of some fungi, and is the second most abundant natural polysaccharide after cellulose. Various advantageous characteristic of CS have been demonstrated, including biocompatibility (Richardson et al., 1999), biodegradability, low toxicity (Kean and Thanou, 2010), cell affinity (Gong et al., 2000), antimicrobial properties (Rabea et al., 2003), and antioxidant behavior (Kim and Thomas, 2007). The cationic nature of CS results in electrostatic interactions between the positively charged CS and negatively charged

\footnotetext{
* Corresponding author: Hsien-Tsung Wu - E-mail: stwu@mail.mcut.edu.tw
} 
mucosal surfaces, and thus promotes transmucosal drugs absorption via a transient widening of the tight junctions between epithelial cells (FernándezUrrusuno et al., 1999). Therefore, this excellent material has been applied in many fields, particularly in medicine and pharmacy for the development of sustained or controlled-release drug delivery systems (Nunthanid et al., 2004; Reverchon and Antonacci, 2007; Li et al., 2014), and was included in the United States Pharmacopoeia in 2011.

Several techniques can be used to prepare polymeric microparticles for controlled drug delivery, for example, solvent evaporation (Takenaka et al., 1982), spray drying (Nunthanid et al., 2004), and ionotropic gelation (Sadeghi et al., 2008). However, these conventional processes have some drawbacks, such as the high temperatures required, the limited size control during spray drying, and time-consuming post-treatment steps required, such as centrifugation and freeze drying, to obtain CS in a dry powder form. Supercritical fluid technology has been proposed as an alternative to these conventional techniques for the production of micro- and nano-sized particles. The supercritical anti-solvent (SAS) process has been previously used to produce microparticles of TPH (Subra et al., 2005; Franceschi et al., 2008) using a mixture of ethanol and dichloromethane as solvent. The effects of pressure, temperature, TPH concentration in the solution and solution flow rate on particle size, size distribution and morphology were investigated. Padrela et al. (2014) used the supercritical enhanced atomization (SEA) process with tetrahydrofuran as solvent to prepare TPH co-crystals with different coformers. The results showed that the low-solubility coformers generated TPH cocrystals with a slow dissolution rate, whereas the use of highly soluble coformers produced faster-dissolving $\mathrm{TPH}$ co-crystals. However, CS is soluble in acidic aqueous solution, and aqueous solvents are difficult to use in the SAS processes owing to the poor solubility of $\mathrm{ScCO}_{2}$ in water.

Supercritical assisted atomization (SAA) involves two atomization steps: pneumatic atomization, induced by the pressure drop at the nozzle outlet, and decompressive atomization, generated by rapidly delivering $\mathrm{CO}_{2}$ inside the primary droplets. The microparticles were produced by the evaporation of solvent and supersaturation of solute in the liquid droplets. SAA can be used with both aqueous and organic solvents, and is a stable, flexible and easily scalable process for particle formation (Reverchon etal., 2006). SAA has also been proposed for the production of composite materials, such as coprecipitation of ampicillin trihydrate/CS and trypsin/chitosan (Reverchon and Antonacci, 2007; Shen et al., 2015) and CS microparticles loaded with magnetite $\left(\mathrm{Fe}_{3} \mathrm{O}_{4}\right)$ nanoparticles (Adami et al., 2012). The prolonged drug release of an SAA coprecipitate could be produced by $\mathrm{CS}$ and AMP dissolved in an aqueous solution of 1\% acetic acid (Reverchon and Antonacci, 2007). Moreover, the encapsulation efficiency of the $\mathrm{Fe}_{3} \mathrm{O}_{4}+$ CS composite particles mainly depends on the stability of the starting suspensions of $\mathrm{Fe}_{3} \mathrm{O}_{4}$ nanoparticles in polymer-solvent solutions. A smaller percentage of $\mathrm{Fe}_{3} \mathrm{O}_{4}$ nanoparticles was loaded (with a good stability for the starting suspensions), resulting in a higher encapsulation efficiency for the SAA composite microparticles (Adami et al., 2012). Therefore, the aim of this study was to evaluate the feasibility of using the SAA process for preparation of TPH-CS composite microparticles. The formulations were characterized by a variety of experimental techniques in order to investigate the particle morphology and drug release kinetics in a buffer solution for controlled drug release systems. The precipitation experiments on TPH-CS composite particles in this study were conducted with various concentrations of polymer solution (C) and with mass ratios of CS to TPH $(Z=\mathrm{CS} / \mathrm{TPH})$ using saturator temperatures ranging from $313.2 \mathrm{~K}$ to 353.2 $\mathrm{K}$. The collected samples were analyzed using a field emission scanning electron microscope (FESEM), and the particle size distributions and the mean particle sizes were then determined using a dynamic light scattering (DLS) particle analyzer. In addition, controlled drug release models were estimated by fitting the experimental results from drug dissolution tests. The mechanisms of the controlled drug release are then discussed based on these results.

\section{MATERIALS AND METHODS}

\section{Materials}

Low molecular weight chitosan $(>75 \%$ degree of deacetylation, $190-310 \mathrm{kDa}$ ) was supplied by Aldrich, USA. Acetic acid (99.9\% purity, HPLC grade) was purchased from Acros, USA. Theophylline (MW = 180.16, 99.9\% purity) was purchased from Sigma Aldrich. Carbon dioxide (99.9\% purity) and nitrogen (99.9\% purity) were purchased from Yung-Ping Gas Co., Taiwan. These chemicals were used without further purification.

\section{Apparatus and operation}

A schematic diagram of the supercritical assisted atomization (SAA) apparatus has been detailed elsewhere ( $\mathrm{Wu}$ and Yang, 2011). The apparatus consists of three main chambers (the saturator, precipitator, and separator) and three feeding lines (the TPH-CS solution, $\mathrm{CO}_{2}$ and $\mathrm{N}_{2}$ ). Two high-pressure liquid pumps were used to deliver $\mathrm{CO}_{2}$ and the TPH$\mathrm{CS}$ solution. The $\mathrm{N}_{2}$ flow was controlled using a mass controller from a cylinder, heated in an electric heat exchanger, and then sent to the precipitator to assist in 
the evaporation of the liquid droplets.

The experimental procedure was as follows. The saturator temperature $\left(T_{S}\right)$ and volumetric flow rate of $\mathrm{CO}_{2}$ were at pre-set values. The precipitator temperature and $\mathrm{N}_{2}$ flow rate were $388.2 \mathrm{~K}$ and 0.8 $\mathrm{Nm}^{3} / \mathrm{h}$, respectively. As a steady state was achieved, the TPH-CS solution was introduced into the saturator via a pre-heating water bath at a flow rate of $4 \mathrm{ml} / \mathrm{min}$. The $\mathrm{CO}_{2}$ mixture that was dissolved in the chitosan solution and obtained by the saturator was sprayed through an injection nozzle to atomize the liquid droplets in the precipitator. After contact of the droplet solution with the heated $\mathrm{N}_{2}$ and the resulting evaporation of solvent from the droplets, the TPH-CS composite particles formed due to the supersaturation of the solute. The collected samples were removed from the precipitator and observed using FESEM (model 6500, JEOL, Japan). The particle size distribution (PSD) and the mean size (the mass-weighted mean sizes, $d_{43}$ ) of the TPH-CS composite particles were determined using a DLS particle analyzer (Zetasizer Nano ZS90, Malvern, UK). Before analysis, the TPH-CS composite particles were suspended in petroleum ether and sonicated for 10 min. All precipitation experiments were performed at least in triplicate and used to calculate the mean values with a standard deviation for each set of conditions. The average yield of the TPH-CS composite particles in the SAA process was $80 \%$. The arithmetic and mass-weighted mean particle diameters, $d_{n o}$ and $d_{4,3}$, respectively, were calculated from the equations $d_{n o}$, $\Sigma d / N$ and $d_{43}=\Sigma d_{i}^{4} \Sigma d_{i}^{3}$, where $N$ represents the number of particles. The polydispersity index (PDI; PDI $=d_{4,3}$ $d_{n o}$ ) characterized the particle size distribution.

\section{Solid-state characterization}

$\mathrm{X}$-ray diffraction (XRD) patterns of the product powders were recorded using an X'Pert Pro X-ray powder diffractometer (PANalytical, Netherlands). The scanning region of the diffraction angle (2) ranged from $5^{\circ}$ to $50^{\circ}$ at a scan rate of $0.02^{\circ} / \mathrm{s}$. Thermograms of the particle samples were determined using a differential scanning calorimeter (DSC, Q-20, TA Instruments, USA). Samples of 3-10 mg were placed in aluminum pans, which were then sealed and heated from $298.2 \mathrm{~K}$ to $553.2 \mathrm{~K}$ at a rate of $10 \mathrm{~K} / \mathrm{min}$ in a $\mathrm{N}_{2}$ atmosphere. Infrared (IR) spectra of the samples were recorded using a Fourier transform infrared (FTIR) spectrophotometer (Spectrum 100, Perkin Elmer, USA) using the $\mathrm{KBr}$ pellet method. The IR spectra were recorded from 4000 to $400 \mathrm{~cm}^{-1}$.

\section{Drug release experiments}

An accurately weighted $5 \mathrm{mg}$ sample of TPH-CS composite microparticles was suspended in $20 \mathrm{~mL}$ water and ultrasonicated for $2 \mathrm{~h}$ to completely dissolve TPH. The dissolved sample was centrifuged at 10,000 rpm for $10 \mathrm{~min}$ and the supernatant was filtrated through a $0.45 \mu \mathrm{m}$ membrane filter. The amount of TPH in the filtered solution was determined with a UV spectrophotometer (JASCO UV IDE-505, Japan) at a wavelength of $273 \mathrm{~nm}$. The drug content $(\%, w / w$ $\mathrm{TPH})$ of the composite particles was determined by calculating the mass ratio between the weight of TPH in the filtered solution and that of the original TPH-CS composite particles. All measurements were carried out in triplicate $(n=3)$.

The in vitro dissolution reported by Reverchon and Antonacci (2007) was slightly modified as follows: the dissolution tests were performed by using USP apparatus 1 (basket method, DT6, Shin Kwang, Taiwan). The tablets (6 $\mathrm{mm}$ in diameter) were prepared by compression using a Carver laboratory press (Model 4350, USA) at 2 tones force for $5 \mathrm{~min}$. Each tablet contained $10 \mathrm{mg}$ TPH equivalent powder. Dissolution tests of the as-received TPH and TPHCS composite particles were performed in $500 \mathrm{~mL}$ of phosphate buffer solution (PBS, $\mathrm{pH}$ 7.2) maintained at a temperature of $310.2 \pm 0.5 \mathrm{~K}$. The rotation speed of the basket was set at $50 \mathrm{rpm}$. At appropriate time intervals, $5 \mathrm{~mL}$ of the dissolution medium was withdrawn and immediately replaced with fresh medium. The amount of dissolved TPH was analyzed using a UV spectrophotometer at $273 \mathrm{~nm}$. All measurements were carried out in triplicate $(n=3)$. The dissolution profiles of the as-received TPH and composite particles with different $\mathrm{CS} / \mathrm{TPH}$ ratios were used to understand the drug release kinetics.

\section{RESULTS AND DISCUSSION}

\section{Characterization of TPH-CS composite particles}

Since chitosan is soluble in acidic aqueous solutions, $1.2 \%(\mathrm{v} / \mathrm{v})$ aqueous acetic acid was used in this study. According to a previous study (Wu et al., 2015), mixing the same volumes of ethanol and $1.2 \%(\mathrm{v} / \mathrm{v})$ aqueous acetic acid (denoted as $50 \%(\mathrm{v} / \mathrm{v})$ aqueous ethanol) as a solvent could reduce the CS particle sizes compared to using $1.2 \%(\mathrm{w} / \mathrm{v})$ aqueous acetic acid as a solvent; this is because decompressive atomization was improved by the increased solubility of $\mathrm{CO}_{2}$ in the liquid phase. Therefore, in this study, $50 \%(\mathrm{v} / \mathrm{v})$ aqueous ethanol was used as a solvent during the SAA process to prepare TPH-CS composite particles. The optimal precipitation parameters of the SAA process were also taken from this previous study (Wu et al., 2015): saturator temperature $\left(T_{S}\right)$ of $353.2 \mathrm{~K}$, CS concentration of the TPH-CS solution $\left(C_{C S}\right)$ of $1 \mathrm{mg} /$ $\mathrm{mL}$, and $\mathrm{CO}_{2}$ to solution flow ratio of 1.8. The effect of CS/TPH mass ratios $(Z=\mathrm{CS} / \mathrm{TPH})$ used during the SAA process on TPH-CS composite particle size was examined over a range of $1-10$, as shown in Table 1 (runs \#1 to \#5). 
Table 1. Experimental conditions and results of the TPH-CS composite particles.

\begin{tabular}{|c|c|c|c|c|c|c|c|c|c|}
\hline \multirow{2}{*}{ Run } & \multirow{2}{*}{$\mathbf{Z}^{\mathbf{a}}$} & $\mathrm{C}_{\mathrm{CS}}^{\mathrm{b}}$ & $\mathrm{C}_{\mathrm{TPH}}{ }^{\mathrm{c}}$ & \multirow{2}{*}{$\mathbf{T s}^{\mathrm{d}}$} & $d_{n o}{ }^{e}$ & $d_{4,3}{ }^{f}$ & \multirow{2}{*}{$\begin{array}{c}\text { PDI } \\
\mathbf{d}_{4,3} / \mathbf{d}_{\mathrm{n} 0}\end{array}$} & \multirow{2}{*}{$\begin{array}{c}\text { Drug cont. }^{\mathrm{g}} \\
(\%)\end{array}$} & \multirow{2}{*}{$\begin{array}{l}t_{63.2 \%}{ }^{h} \\
\text { (min) }\end{array}$} \\
\hline & & \multicolumn{2}{|c|}{$(\mathrm{mg} / \mathrm{mL})$} & & \multicolumn{2}{|c|}{ (nm) } & & & \\
\hline 1 & 1 & 1 & 1 & 353.2 & $608 \pm 26$ & $696 \pm 8$ & 1.14 & $49.2 \pm 0.4$ & 64 \\
\hline 2 & 3 & 1 & 0.33 & 353.2 & $510 \pm 3$ & $632 \pm 32$ & 1.24 & $23.7 \pm 0.4$ & 134 \\
\hline 3 & 5 & 1 & 0.2 & 353.2 & $469 \pm 55$ & $581 \pm 10$ & 1.24 & $15.8 \pm 0.5$ & 216 \\
\hline 4 & 7 & 1 & 0.14 & 353.2 & $454 \pm 23$ & $493 \pm 38$ & 1.09 & $12.9 \pm 0.2$ & 274 \\
\hline 5 & 10 & 1 & 0.1 & 353.2 & $438 \pm 6$ & $468 \pm 5$ & 1.07 & $9.3 \pm 0.3$ & 341 \\
\hline 6 & 5 & 1 & 0.2 & 313.2 & $527 \pm 27$ & $676 \pm 21$ & 1.28 & $16.1 \pm 0.9$ & 187 \\
\hline 7 & 5 & 1 & 0.2 & 333.2 & $502 \pm 33$ & $635 \pm 23$ & 1.25 & $16.6 \pm 0.9$ & 192 \\
\hline 8 & 5 & 0.5 & 0.1 & 353.2 & $450 \pm 18$ & $534 \pm 23$ & 1.19 & $16.3 \pm 0.1$ & 226 \\
\hline 9 & 5 & 1.5 & 0.3 & 353.2 & $482 \pm 18$ & $604 \pm 14$ & 1.26 & $16.5 \pm 0.9$ & 198 \\
\hline
\end{tabular}

the chitosan/theophylline mass ratio.

${ }^{b}$ the concentration of chitosan in the feed solution.

${ }^{c}$ the concentration of theophylline in the feed solution.

d the saturator temperature.

${ }^{\mathrm{e}} d=\left(\sum_{\mathrm{i}=0}^{\mathrm{N}} \mathrm{Di}\right) / \mathrm{N}$, the arithmetic mean size.

${ }^{\mathrm{f}} d_{43}=\left(\sum_{\mathrm{i}=0}^{\mathrm{N}} \mathrm{Di}^{4}\right) /\left(\sum_{\mathrm{i}=0}^{\mathrm{N}} \mathrm{Di}^{3}\right)$, the mass-weighted mean size.

$\mathrm{g}$ the drug content in composite particles $(\%)$.

${ }^{\mathrm{h}}$ the estimated time of $63.2 \%$ drug released from the Weibull model.

FESEM images of the composite particles with different CS/TPH ratios and their PSDs are presented in Fig. 1 and Fig. 2, respectively. The crystalline TPH in Fig. 1 gradually disappears as the CS content in the TPH-CS composite microparticles (high $Z$-value samples) increases. Moreover, the morphologies of high $Z$-value TPH-CS composite microparticles are similar to those observed in a previous study (Wu et al., 2015) of SAA-CS with sub-micrometer spherical CS particles and narrow PSDs, as shown in Fig. 2. Figures $1 \mathrm{c}$ and $1 \mathrm{f}$ show a comparison of TPH-CS composite microparticles and a physical mixture (PM) at the same TPH content $(Z=5)$. It was observed that the PM showed separation of CS and TPH, whereas the TPH-CS composite microparticles were uniformly dispersed or a solid dispersion of CS and TPH.

XRD, DSC and FTIR analyses of the as-received TPH and SAA-processed TPH-CS composite particles are shown in Fig. 3, Fig. 4, and Fig. 5, respectively. XRD analysis showed that the as-received TPH had a crystalline structure, whereas the as-received CS was a semi-crystalline solid due to a halo diffraction pattern.

A.

B. C.

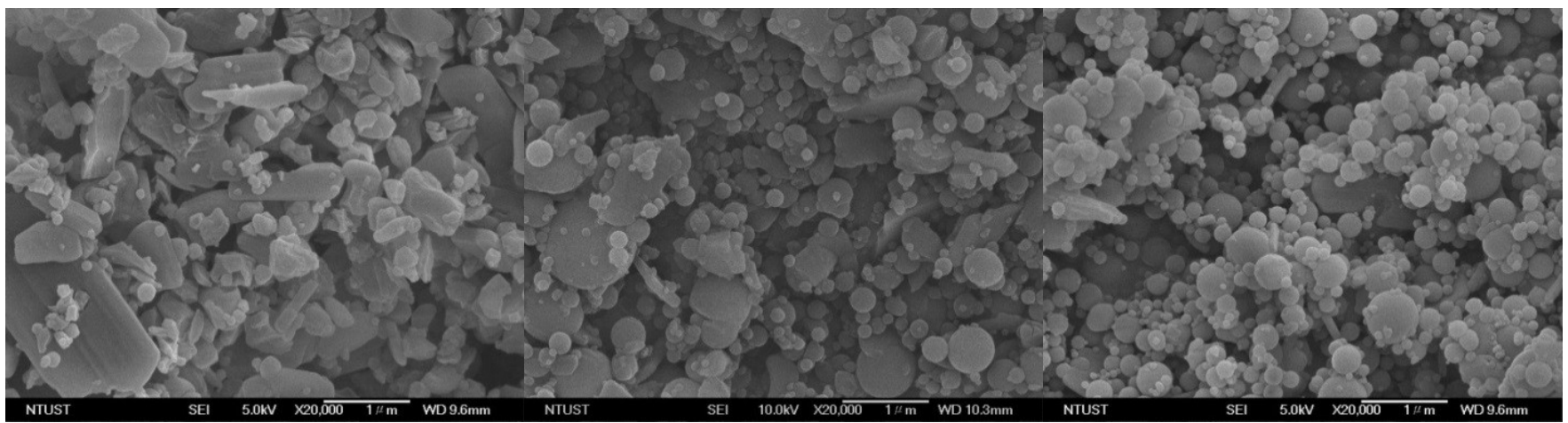

D.

E.

F.

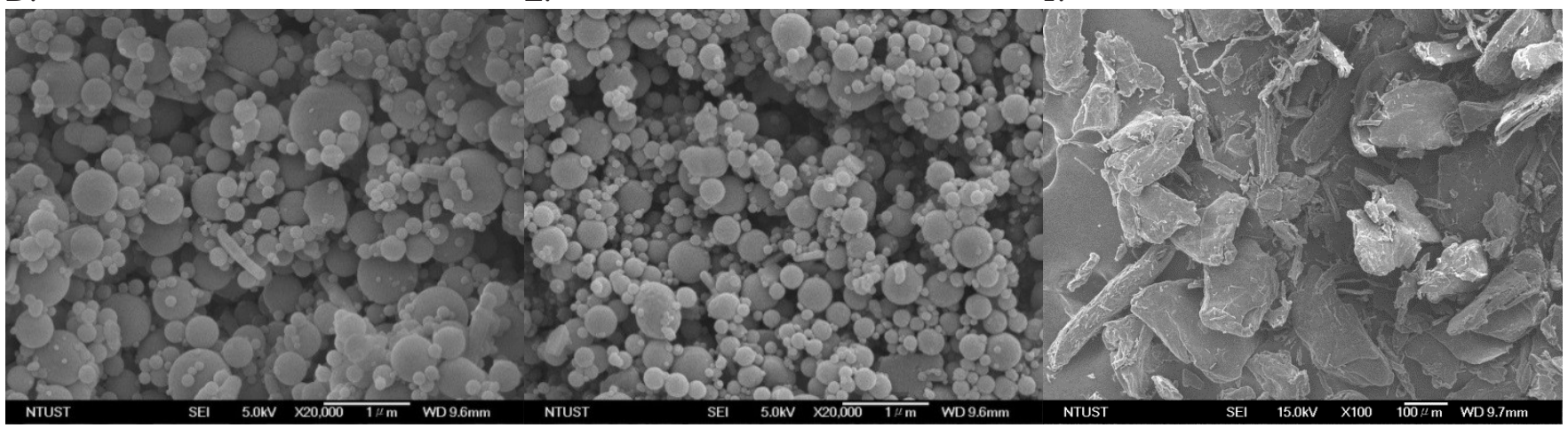

Figure 1. FESEM images of the TPH-CS composite particles produced using SAA at different CS/TPH ratio (Z): (a) $Z=1$, (b) $Z=3$, (c) $Z=5$, (d) $Z=7$, (e) $Z=10$, (f) $Z=5$ (PM). 


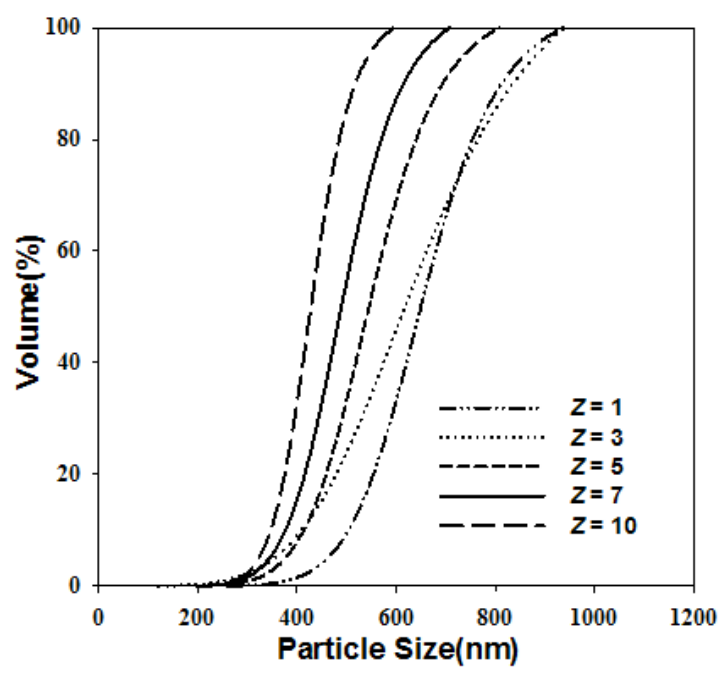

Figure 2. PSDs of the TPH-CS composite particles produced using SAA at different CS/ TPH ratios (Z).

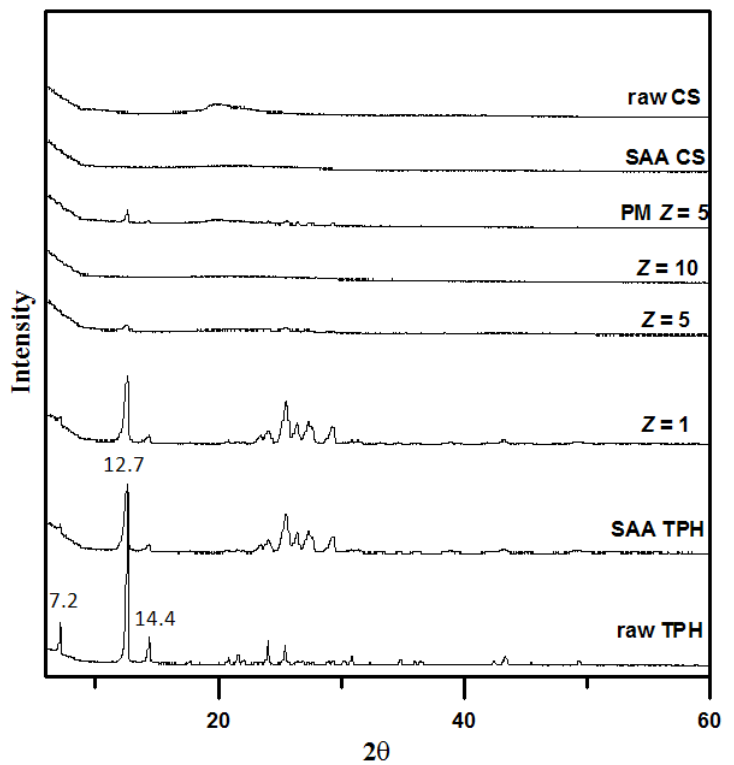

Figure 3. The XRD analyses of the TPH-CS composite particles produced using SAA at different $\mathrm{CS} / \mathrm{TPH}$ ratios $(Z)$.

The as-received TPH showed the characteristics of the anhydrous form I with characteristic XRD peaks at 7.2, 12.7 and $14.4^{\circ}$ (Franceschi et al., 2008; Airaksinen et al., 2004; Fini et al., 2011; Phadnis and Suryanarayanan, 1997). The PM sample $(Z=5)$ and SAA-processed TPHCS composite particles showed the same characteristic peaks with no difference in crystalline form. When the content of CS in TPH-CS composite particles was increased (high $Z$ value), the diffraction patterns showed an amorphous halo with no observable crystalline peaks.

The polymorphs were further confirmed by DSC results (Fig. 4). The melting points of the anhydrous I-theophylline and SAA-processed TPH particles (SAA-TPH) were found to be $544.2-547.2 \mathrm{~K}$. The SAA-TPH particles showed a slight lowering of the

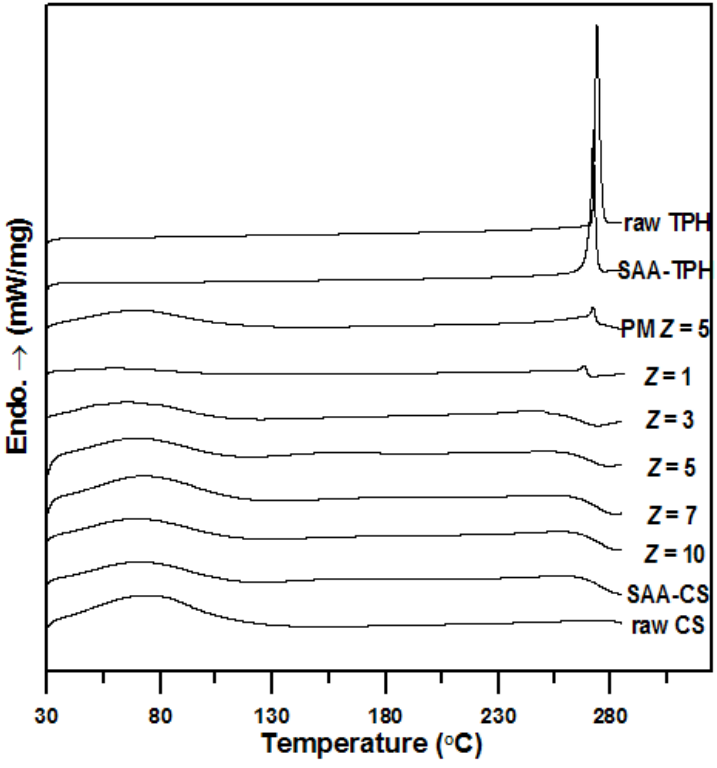

Figure 4. DSC analyses of TPH-CS composite particles produced using SAA at different $\mathrm{CS} / \mathrm{TPH}$ ratios $(Z)$.

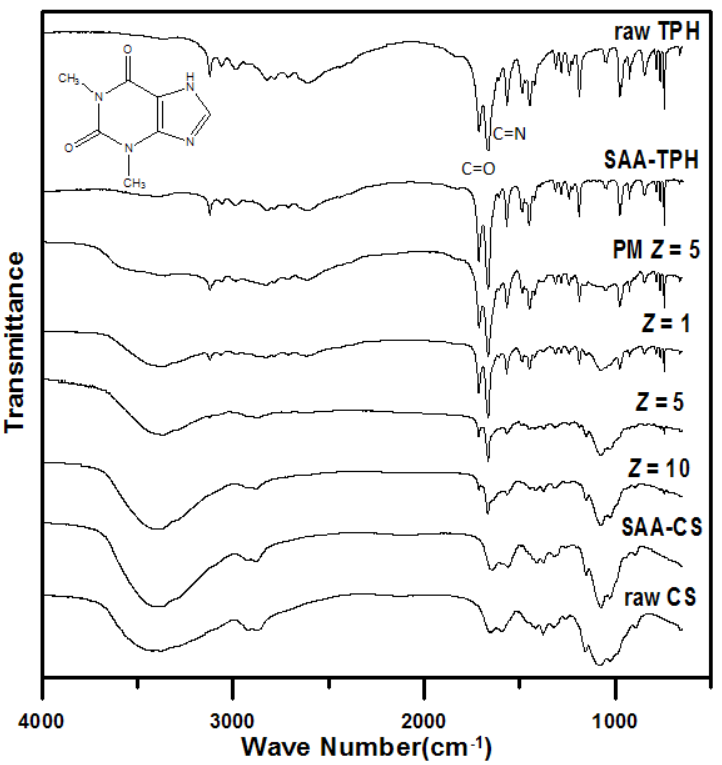

Figure 5. FTIR analyses of TPH-CS composite particles produced using SAA at different $\mathrm{CS} / \mathrm{TPH}$ ratios $(Z)$.

melting temperature and enthalpy of fusion (from $162.4 \mathrm{~J} / \mathrm{g}$ to $140.2 \mathrm{~J} / \mathrm{g}$ ), attributed to a reduction in the SAA-TPH particle size (Franceschi et al., 2008). The melting peak of the TPH-CS composite particles $(25 \%, Z=3)$ was not detected due to dissolution in the molten carrier. Increasing the TPH content in the TPHCS composite particles $(50 \%, Z=1)$, a deformation of the baseline and further lowering of the drug melting point suggested that TPH reaches its saturation and the drug existed in its crystalline form as well as in a dissolved state. Similar results have been reported in the literature (Fini et al., 2011; Passerini et al., 2010). 
FTIR spectra (Fig. 5) showed that the as-received TPH had characteristic peaks at $1718 \mathrm{~cm}^{-1}$ and 1665 $\mathrm{cm}^{-1}$ assigned to the asymmetric and symmetric stretching vibrations, respectively, of the two carbonyl groups $(\mathrm{C}=\mathrm{O})$, and at $1567 \mathrm{~cm}^{-1}$, assigned to the $\mathrm{C}=\mathrm{N}$ stretching vibrations. (Fini et al., 2011). The PM sample $(Z=5)$ showed the same characteristic peaks as those of the as-received TPH with the typical anhydrate form I. SAA-processed TPH-CS composite particles did not show new peaks, indicating an absence of intermolecular interactions between TPH and CS. Similar results have been reported in TPHCS composite particles produced using a spray drying process (Nunthanid et al., 2007; Puttipipatkhachorn et al., 2001).

\section{Effects of mass ratio and composite particle size on dissolution}

The drug content of the TPH-CS composite particles at different mass ratios $(Z)$ produced using SAA is presented in Table 1. The results show that the samples had nearly the same drug content as the feed ratio of TPH, showing that the TPH-CS composite particles formed a solid dispersion of CS and TPH. We also repeatedly measured the drug content of TPHCS composite particles stored at $60 \% \mathrm{RH}$ and room temperature for 12 months to verify the stability of TPH-CS composite particles. The data are follows: $47.5 \pm 0.7 \%$ (run $1,49.2 \pm 0.5 \%$ in Table 1 ), $23.6 \pm$ $0.3 \%$ (run $2,23.7 \pm 0.4 \%$ in Table 1 ), $15.9 \pm 0.3 \%$ (run 3, $15.8 \pm 0.5 \%$ in Table 1), and $9.2 \pm 0.3 \%$ (run $5,9.3 \pm 0.3 \%$ in Table 1). The results showed almost no changes in the drug content of TPH in the composite particles and indicated the good stability of the composite particles during the storage time of 12 months.

Drug release analyses were carried out using powder produced at $Z=1-10$ to verify the effect of the CS/TPH mass ratio on the dissolution rate. Fig. 6 shows the TPH release profiles from tablets containing co-precipitated microparticles over a given time interval (Fig. 6a) and the initial $60 \%$ drug release data (Fig. 6b). The as-received TPH is not shown in Fig. 6; it has a very fast dissolution rate as it is a highly watersoluble drug. Incorporation of the CS carrier retarded the dissolution of TPH as compared to TPH alone. The size of the composite particles and the time for $63.2 \%$ drug released $\left(t_{63.2 \%}\right)$ at different $\mathrm{CS} / \mathrm{TPH}$ ratios $(Z)$ are also presented in Table 1 . It can be seen that different $Z$ values have an insignificant effect on the mean size of the composite particles, as presented in a study of ampicillin-CS composite particles reported by Reverchon and Antonacci (2007). However, the $\mathrm{CS} / \mathrm{TPH}$ ratio had a crucial influence on the drug dissolution rate of the TPH-CS composite particles. The TPH dissolution rates decreased with increasing $Z$
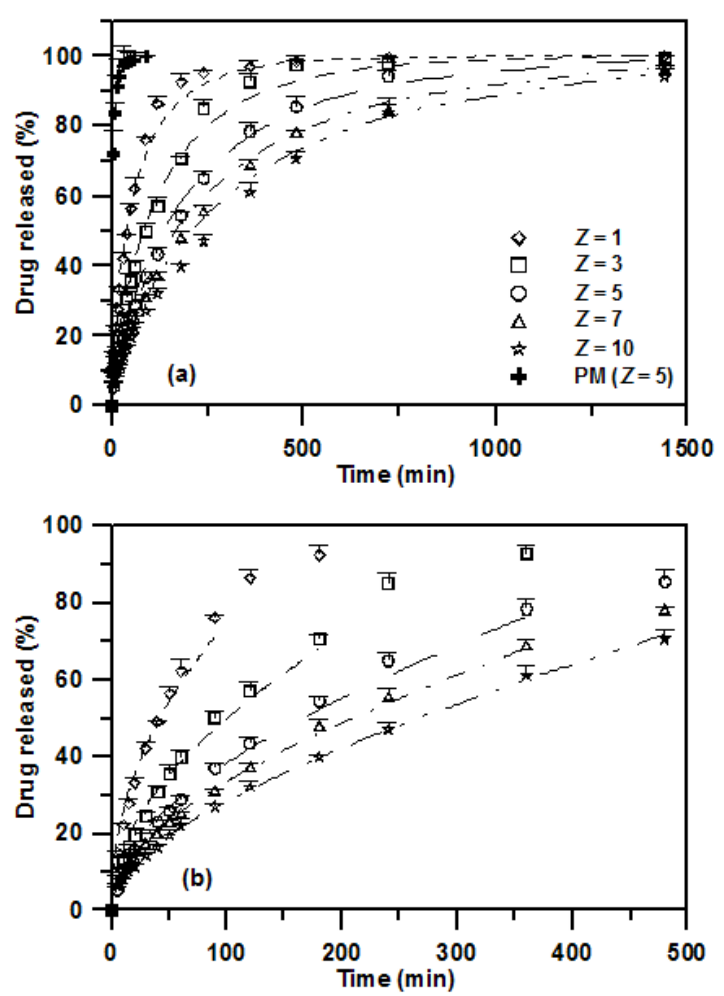

Figure 6. Dissolution profiles of the TPH-CS composite particles produced using SAA at different $\mathrm{CS} / \mathrm{TPH}$ ratios $(Z)$. (a) Complete dissolution data and the calculated values (lines) obtained from the Weibull model. (b) Initial 60\% drug release data and the calculated values (lines) obtained from the PeppasSahlin model.

values. The sample with $Z=10$ with a sustained release of $63.2 \%$ TPH was dissolved at $341 \mathrm{~min}$, which could be due to the polymer swelling or slow relaxation rate of the CS carrier. The sustained drug release was attributed mainly to the gel formation of the SAAprocessed TPH-CS composite particles following absorption of water from the dissolution medium. Similar results were obtained for co-precipitates of TPH and chitosan acetate prepared using spray drying (Nunthanid et al., 2007).

The particle size of the SAA-processed material can be manipulated by changing the saturator temperature and concentration of the feed solution (Wu et al., 2015). Therefore, these factors were varied during SAA to prepare TPH-CS composite particles with different sizes but the same composition $(Z=5)$. The mean size of the composite particles $\left(d_{43}\right)$ decreased at high saturator temperature and ranged from $676 \mathrm{~nm}$ to 581 $\mathrm{nm}$ (Table 1, runs \#3, \#6 and \#7) at different saturator temperatures. The composite particle size could be further reduced by decreasing the concentration of feed solution (runs \#3, \#8 and \#9). The $d_{4,3}$ value of the composite particles at different concentrations of feed solution varied from $604 \mathrm{~nm}$ to $534 \mathrm{~nm}$. Thus, no effect of the TPH-CS composite particle size on the 
dissolution rate was observed in the present study. We attribute this to the small range of particle sizes that could be produced under the experimental conditions used.

\section{Drug release models}

Modelling of drug release profiles can correlate and predict the effect of the drug dosage and characterize the different release mechanisms involved. This is important in the design of drug delivery systems. The Weibull distribution can be successfully applied to most types of drug dissolution curves (Costa and Lobo, 2001). It expresses the accumulated fraction of drug, $m$, in a solution at time, $t$, by:

$\mathrm{m}=1-\exp \left[\frac{-\mathrm{t}^{\mathrm{b}}}{\mathrm{a}}\right]$

where $a$ is defined as the time scale of the process and $b$ is the shape parameter. A linear relation can be obtained for a log-log plot of $-\ln (1-\mathrm{m})$ versus time $t$. The value of $b$ is obtained from the slope of the line and $a$ is estimated from the ordinate value $(1 / a)$ at $t$ $=1$. The parameter $a$ can be replaced by the specific dissolution time, $T_{d}=(a)^{\wedge}(-1 / b)$. Since $-\ln (1-m)=1$ is equivalent to $m=0.632, T_{d}$ represents the time required to dissolve or release $63.2 \%$ of the drug present in the dosage form. The correlated results of the dissolution profiles of the TPH-CS composite particles are shown in Table 2, which correspond to the marked curves in Fig. 6a. Coefficients of determination $\left(R^{2}\right)$ for the drug dissolution data were $>0.98$. However, the Weibull equation is an empirical model, not deducted from any fundament kinetic theory.

To understand the drug release mechanism, the first $60 \%$ of the drug release data was fitted using the Korsmeyer-Peppas model, which is a semi-empirical model based on a power-law expression to analyze the drug release from a swelling polymer system (Ritger and Peppas, 1987), as follows:
$\frac{\mathrm{M}_{\mathrm{t}}}{\mathrm{M}_{\infty}}=\mathrm{kt}^{\mathrm{n}}$

where $M_{t}$ and $M_{\infty}$ are the cumulative amounts of drug released at any specific time $t$ and infinite time $\infty$, respectively, $k$ is the Korsmeyer-Peppas release rate constant, $n$ is the diffusion exponent, and $M_{t} / M_{\infty}$ is the fraction of the drug release at time $t$. Non-linear regression analysis was performed on curves obtained from data fitting to the Korsmeyer-Peppas model to obtain the rate constants and $n$ values, which might be indicative of the mechanism of drug release for different geometries. For a cylindrical sample, the $n$ values are 0.45 and 0.89 for diffusion and "polymer swelling" controlled drug delivery, respectively. If $0.45<n<0.89$, the drug release mechanism follows anomalous (non-Fickian) diffusion, and if $n=0.89$, the drug release could be purely due to polymer swelling (Case-II transport) (Ritger and Peppas, 1987). The correlated results of controlled drug release data fitted using the Korsmeyer-Peppas model are shown in Table 2. The $n$ values ranged from 0.477 to 0.580 , suggesting anomalous or non-Fickian diffusion. This indicates that the drug release from these composite particles could result from a combination of both drug diffusion and dissolution of the polymer.

To distinguish the drug release mechanism, we further used the Peppas-Sahlin model shown in Eq. (3). In this model, the first term of the right-hand side is the contribution from Fickian diffusion and the second term is related to polymer relaxation (Peppas and Sahlin, 1989)

$\frac{M_{t}}{M_{\infty}}=k_{1} t^{n}+k_{2} t^{2 n}$

where $k_{1}$ and $k_{2}$ are the diffusion and relaxation rate constants, respectively, and $n$ is the diffusion exponent. The value of $n$ was 0.45 because the tablets used in this study had an aspect ratio (diameter/thickness) around

Table 2. Correlated results of the in vitro dissolution profiles of the TPH-CS composite particles.

\begin{tabular}{|c|c|c|c|c|c|c|c|c|c|c|c|c|c|}
\hline \multirow{2}{*}{ Run } & \multirow{2}{*}{$\mathbf{Z}^{\mathbf{a}}$} & \multirow{2}{*}{$\begin{array}{c}\mathrm{C}_{\mathrm{TPH}} \\
(\mathrm{mg} / \mathrm{mL})\end{array}$} & \multirow{2}{*}{$\begin{array}{l}\mathbf{T}_{\mathbf{s}} \\
\mathbf{K}\end{array}$} & \multicolumn{4}{|c|}{ Weibull $^{b}$} & \multicolumn{3}{|c|}{ Korsemeyer-Peppas $^{\mathrm{c}}$} & \multicolumn{3}{|c|}{ Peppas-Sahlind $^{d}$} \\
\hline & & & & $\mathbf{a}$ & b & $\mathbf{r}^{2}$ & $\mathbf{T}_{\mathbf{d}}{ }^{\mathbf{e}}$ & $\mathbf{k}$ & $\mathrm{n}$ & $\mathbf{r}^{2}$ & $\mathbf{k}_{1}$ & $\mathbf{k}_{\mathbf{2}}$ & $\mathbf{r}^{2}$ \\
\hline 1 & 1 & 1 & 353.2 & 18.20 & 0.699 & 0.98 & 64 & 8.367 & 0.477 & 0.98 & 9.069 & 0.0468 & 0.98 \\
\hline 2 & 3 & 0.33 & 353.2 & 41.52 & 0.761 & 0.99 & 134 & 4.507 & 0.524 & 0.99 & 5.408 & 0.1120 & 0.99 \\
\hline 3 & 5 & 0.2 & 353.2 & 61.69 & 0.767 & 0.99 & 216 & 3.287 & 0.535 & 0.99 & 4.079 & 0.0938 & 0.99 \\
\hline 4 & 7 & 0.14 & 353.2 & 62.05 & 0.736 & 0.98 & 274 & 2.743 & 0.546 & 0.99 & 3.444 & 0.0971 & 0.99 \\
\hline 5 & 10 & 0.1 & 353.2 & 78.28 & 0.748 & 0.98 & 341 & 2.070 & 0.572 & 0.99 & 2.717 & 0.1080 & 0.99 \\
\hline 6 & 5 & 0.2 & 313.2 & 50.08 & 0.748 & 0.98 & 187 & 3.351 & 0.545 & 0.99 & 4.115 & 0.1284 & 0.99 \\
\hline 7 & 5 & 0.2 & 333.2 & 56.96 & 0.769 & 0.99 & 192 & 3.230 & 0.549 & 0.99 & 4.005 & 0.1291 & 0.99 \\
\hline 8 & 5 & 0.1 & 353.2 & 63.12 & 0.765 & 0.99 & 226 & 2.665 & 0.580 & 0.99 & 3.475 & 0.1636 & 0.99 \\
\hline 9 & 5 & 0.3 & 353.2 & 54.99 & 0.758 & 0.99 & 198 & 3.250 & 0.547 & 0.99 & 4.011 & 0.1252 & 0.99 \\
\hline
\end{tabular}

a the CS/TPH mass ratio.

b Weibull: $\mathrm{m}=1-\exp \left[-\mathrm{t}^{\mathrm{b}} / \mathrm{a}\right]$

${ }^{c}$ Korsemeyer-Peppas equation: $\left(\mathrm{M}_{\mathrm{t}} / \mathrm{M}_{\infty}\right)=\mathrm{kt}^{\mathrm{n}}$

${ }^{\mathrm{d}}$ Peppas-Sahlin equation: $\left(\mathrm{M}_{\mathrm{t}} / \mathrm{M}_{\infty}\right)=\mathrm{k}_{1} \mathrm{t}^{\mathrm{n}}+\mathrm{k}_{2} \mathrm{t}^{2 \mathrm{n}},(n=0.45)$.

${ }^{e}$ the time of $63.2 \%$ drug released $(\min ), \mathrm{T}_{\mathrm{d}}=(\mathrm{a})^{-1 / \mathrm{b}}$. 
6 (Peppas and Sahlin, 1989). The fraction of drug release $(F)$ due to the Fickian diffusion mechanism can be calculated according to Eq. (4):

$$
\mathrm{F}=\frac{1}{1+\frac{\mathrm{k}_{2}}{\mathrm{k}_{1}} \mathrm{t}^{\mathrm{n}}}
$$

Non-linear regression analysis was performed on curves obtained from data fitting to the Peppas-Sahlin model to obtain the rate constants, which can indicate the mechanism of drug release in composite particles with different $\mathrm{CS} / \mathrm{TPH}$ ratios. The correlated results of controlled drug release data fitted using the PeppasSahlin model are shown in Table 2 and correspond to the marked curves in Fig. 6b. The $R^{2}$ values of the data fitting were $>0.98$. This confirmed that the in vitro dissolution data of the TPH-CS composite particles produced by SAA could be effectively fit using the Peppas-Sahlin model. The $F$ values for Fickian diffusion were calculated using Eq. (4) and are presented in Fig. 7. As $Z$ increased, the contribution of the release mechanism related to relaxation of the polymer increased with respect to the Fickian mechanism. Similar results were also observed for the physical mixture of TPH and CS-anionic polymer ( $\mathrm{Li}$ et al., 2014) and ampicillin trihydrate-CS composite particles prepared by SAA (Reverchon and Antonacci, 2007).

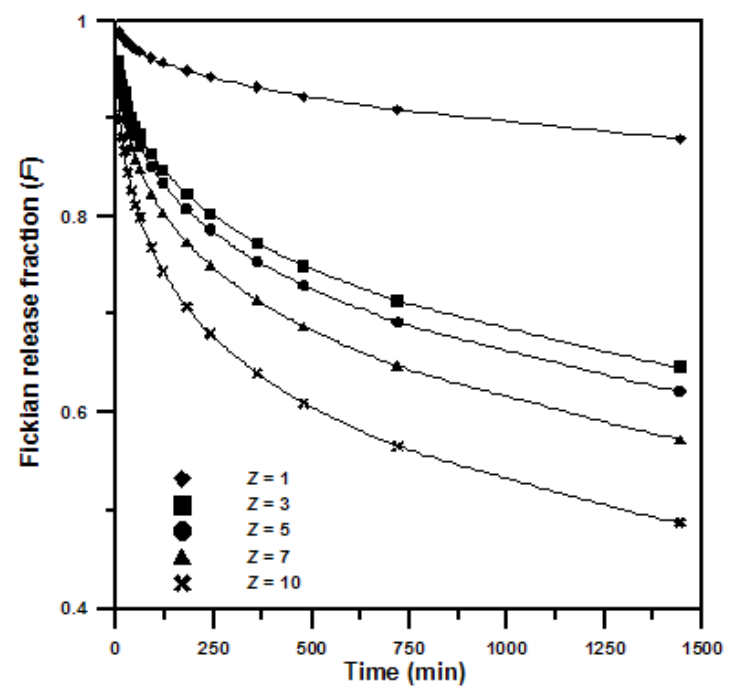

Figure 7. The Fickian release fraction of TPH-CS composite particles produced using SAA at different $\mathrm{CS} / \mathrm{TPH}$ ratios $(Z)$.

\section{CONCLUSIONS}

TPH-CS composite microparticles were successfully prepared using SAA with 50\% (v/v) aqueous ethanol as a solvent, and $\mathrm{CO}_{2}$ as a spraying medium. According to XRD, DSC, and FTIR results, the crystal forms of the SAA-treated TPH and the asreceived TPH were the same. The effect of the mass ratio of $\mathrm{CS}$ to $\mathrm{TPH}$ on the in vitro release of TPH was investigated using dissolution tests. These tests showed that the $t_{63.2 \%}$ of the composite particles was significantly extended compared to pure TPH and the physical mixture of TPH-CS due of the incorporation of water insoluble CS carrier in composite particles. The Peppas-Sahlin model correlated well with the in vitro dissolution data of TPH-CS composite particles, and the drug release was the result of both diffusive and relaxation mechanisms. As the mass ratio of $\mathrm{CS}$ to TPH increased, the contribution of the relaxation of the polymer increased with respect to the diffusion of drug in the TPH-CS composite particles.

\section{ACKNOWLEDGEMENT}

The authors gratefully acknowledge the financial support of the Ministry of Science and Technology, Taiwan, through grant no. MOST 104-2221-E131-024.

\section{REFERENCES}

Adami, R., Reverchon, E. Composite polymer$\mathrm{Fe}_{3} \mathrm{O}_{4}$ microparticles for biomedical applications, produced by supercritical assisted atomization. Powder Technology, 218, 102-108 (2012). https:// doi.org/10.1016/j.powtec.2011.11.048

Airaksinen, S., Karjalainen, M., Räsänen, E., Rantanen, J., Yliruusi, J. Comparison of the effects of two drying methods on polymorphism of theophylline. International Journal of Pharmaceutics, 276, 129-141 (2004). https://doi.org/10.1016/j. ijpharm.2004.02.017

Costa, P.J., Lobo, M.S. Modeling and comparison of dissolution profiles. European Journal of Pharmaceutical Sciences, 13, 123-133 (2001). https://doi.org/10.1016/S0928-0987(01)00095-1

Fernández-Urrusuno, R., Calvo,P., Remuñán-López,C., Vila-Jato, J.L., Alonso, M.J. Enhancement of nasal absorption of insulin using chitosan nanoparticles. Pharmaceutical Research, 16, 1576-1581 (1999). https://doi.org/10.1023/A:1018908705446

Fini, A., Cavallari, C., Ospitali, F., Gonzalez-Rodriguez, M.L. Theophylline-loaded compritol microspheres prepared by ultrasound-assisted atomization. Journal of Pharmaceutical Sciences, 100, $743-757$ (2011). https://doi.org/10.1002/jps.22312

Franceschi, E., Kunita, M.H., Tres, M.V., Rubira, A.F., Muniz, E.C., Corazza, M.L., Dariv, C., Ferreira, S.R.S., Oliveira, J.V. Phase behavior and process parameters effects on the characteristics of precipitated theophylline using carbon dioxide as antisolvent. Journal of Supercritical Fluids, 44, 8-20 (2008). https://doi.org/10.1016/j. supflu.2007.09.031 
Gong, H., Zhong, Y., Li, J., Gong, Y., Zhao, N., Zhang, $X$. Studied on never affinity of chitosan-derived materials. Journal ofBiomedical MaterialsResearch, 52, 285-295 (2000). https://doi.org/10.1002/1097$4636(200011) 52: 2 \% 3$ C $285:$ : A I D JBM7\%3E3.0.CO;2-G

Hendeles, L., Weinberger, M., Johnson, G. Monitoring serum theophylline levels. Clinical Pharmacokinetics, 3, 294-312 (1978). https://doi. org/10.2165/00003088-197803040-00003

Kean, T., Thanou, M. Biodegradation, biodistribution and toxicity of chitosan. Advanced Drug Delivery Reviews, 62, 3-11 (2010). https://doi.org/10.1016/j. addr.2009.09.004

Kim, K.W., Thomas, R.L. Antioxidative activity of chitosans with varying molecular weights. Food Chemistry, 101, 308-313 (2007). https://doi. org/10.1016/j.foodchem.2006.01.038

Legendre, B., Randzio, S.L. Transitiometric analysis of solid II/solid I transition in anhydrous theophylline. International Journal of Pharmaceutics, 343, 41-47 (2007). https://doi.org/10.1016/j. ijpharm.2007.04.015

Li, L., Wang, L., Li, J., Jiang, S., Wang, Y., Zhang, X., Ding, J., Yu, T., Mao, S. Insights into the mechanisms of chitosan-anionic polymers-based matrix tablets for extended drug release. International Journal of Pharmaceutics, 476, 253-265 (2014). https://doi. org/10.1016/j.ijpharm.2014.09.057

Lindenberg, M., Kopp, S., Dressman, J.B. Classification of orally administered drugs on the World Health Organization Model list of Essential Medicines according to the biopharmaceutics classification system. European Journal of Pharmaceutics and Biopharmaceutics, 58, 265-78 (2004). https://doi. org/10.1016/j.ejpb.2004.03.001

Nunthanid, J., Laungtana-anan, M., Sriamornsak, P., Limmatvapirat, S., Puttipipatkhachorn, S., Lim, L.Y., Khor, E. Characterization of chitosan acetate as a binder for sustained release tablets. Journal of Controlled Release, 99, 15-26 (2004). https://doi. org/10.1016/j.jconrel.2004.06.008

Padrela, L., Rodrigues, M.A., Tiago, J., Velaga, S.P., Matos, H.A., Azevedo, E.G de. Tuning physicochemical properties of theophylline by co-crystallization using the supercritical fluid enhanced atomization technique. Journal of Supercritical Fluids, 86, 129-136 (2014). https:// doi.org/10.1016/j.supflu.2013.12.011

Passerini, N., Qi, S.,Albertini, B., Grassi, M., Rodriguez, L., Craig, D.Q.M. Solid lipid microparticles produced by spray congealing: influence of the atomizer on microparticle characteristics and mathematical modeling of the drug release. Journal of Pharmaceutical Sciences, 99, 916-931 (2010). https://doi.org/10.1002/jps.21854
Peppas, N.A., Sahlin, J.J. A simple equation for the description of solute release. III. Coupling of diffusion and relaxation. International Journal of Pharmaceutics, 57, 169-172 (1989). https://doi. org/10.1016/0378-5173(89)90306-2

Phadnis, N.V., Suryanarayanan, R. Polymorphism in anhydrous theophylline-implications on the dissolution rate of theophylline tablets. Journal of Pharmaceutical Sciences, 86, 1256-1263 (1997). https://doi.org/10.1021/js9701418

Puttipipatkhachorn, S., Nunthanid, J., Yamamoto, K., Peck, G.E. Drug physical state and drug-polymer interaction on drug release from chitosan matrix films. Journal of Controlled Release, 75, 143-153 (2001). https://doi.org/10.1016/S0168-3659(01)00389-3

Rabea, E.I., Mohamed, E.T.B., Stevens, C.V., Smagghe, G., Steurbaut, W. Chitosan as antimicrobial agent: applications and mode of action. Biomacromolecules, 4, 1457-1465 (2003). https://doi.org/10.1021/bm034130m

Reverchon, E., Adami, R, Caputo, G. Supercritical assisted atomization: performance comparison between laboratory and pilot scale. Journal of Supercritical Fluids, 37, 298-306 (2006). https:// doi.org/10.1016/j.supflu.2006.01.017

Reverchon, E., Antonacci, A. Drug-polymer microparticles produced by supercritical assisted atomization. Biotechnology and Bioengineering, 97, 1626-1637 (2007). https://doi.org/10.1002/bit.21370

Richardson, S.C., Kolbe, H.V., Duncan, R. Potential of low molecular mass chitosan as a DNA delivery system: biocompatibility, body distribution and ability to complex and protect DNA. International Journal of Pharmaceutics, 178, 231-243 (1999). https://doi.org/10.1016/S0378-5173(98)00378-0

Ritger, P.L., Peppas, N.A. A simple equation for description of solute release II. Fickian and anomalous release from swellable devices. Journal of Controlled Release, 5, 37-42 (1987). https://doi. org/10.1016/0168-3659(87)90035-6

Sadeghi, A.M.M., Dorkoosh, F.A., Avadi, M.R., Saadat, P., Rafiee-Tehrani, M., Junginger, H.E. Preparation, characterization and antibacterial activities of chitosan, $N$-trimethyl chitosan (TMC) and $N$-diethylmethyl chitosan (DEMC) nanoparticles loaded with insulin using both the ionotropic gelation and polyelectrolyte complexation methods. International Journal of Pharmaceutics, 355, 299-306 (2008). https://doi. org/10.1016/j.ijpharm.2007.11.052

Shen, Y.-B., Guan, Y.-X., Yao, S.-J. Supercritical fluid assisted production of micrometric powders of the labile trypsin and chitosan/trypsin composite microparticles. International Journal of Pharmaceutics, 489, 226-236 (2015). https://doi. org/10.1016/j.ijpharm.2015.05.004 
Subra. P., Laudani, C.G., Vega-González, A., Reverchon, E. Precipitation and phase behavior of theophylline in solvent-supercritical $\mathrm{CO}_{2}$ mixtures. Journal of Supercritical Fluids, 35, 95-105 (2005). https://doi.org/10.1016/j. supflu.2004.12.010

Takenaka, H., Kawashima, Y., Chikamatsu, Y., Ando, Y. Reactivity and stability of microencapsulated placental alkaline phosphatase. Chemical and Pharmaceutical Bulletin, 30, 695-701 (1982). https://doi.org/10.1248/cpb.30.695

Talukdar, M.M., van den Mooter, G., Augustijns, P., Tjandra-Maga, T., Verbeke, N., Kinget, R. In vivo evaluation of xanthan gum as a potential excipient for oral controlled-release matrix tablet formulation. International Journal of Pharmaceutics, 169,
105-113 (1998). https://doi.org/10.1016/S03785173(98)00112-4

The United States Pharmacopeial Convention: Rockville, MD, USA, 2011; 5361-5365, Second Supplement.

Wu, H.T., Yang, M.W. Precipitation kinetics of PMMA sub-micrometric particles with a supercritical assisted-atomization process. Journal of Supercritical Fluids, 59, 98-107 (2011). https:// doi.org/10.1016/j.supflu.2011.08.001

Wu, H.T., Lee, H.K., Chen, H.C., Chien, L.J. Precipitation kinetics and biological properties of chitosan microparticles produced using supercritical assisted atomization. Chemical Engineering Research and Design, 104, 615-625 (2015). https:// doi.org/10.1016/j.cherd.2015.09.021 